\title{
Transverse Temperature Distributions and Heat Generation Rate in Composite Conductors Subjected to a Constant Thermal Disturbance*
}

\author{
Y. S. Cha \\ Energy Technology Division \\ Argonne National Laboratory \\ 9700 South Cass Avenue \\ Argonne, IL 60439
}

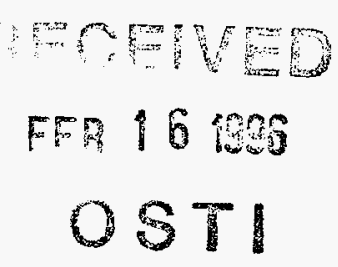

April 1994

Distribution

1. R. W. Weeks

2. R. B. Poeppel

3. R. A. Valentin

DISCLAIMER

4. Authors

5. SA Section

6. B. Baudino

7. F. Y. Fradin

8. H. Drucker

9. S. Lake

\begin{abstract}
This report was prepared as an account of work sponsored by an agency of the United States Government. Neither the United States Government nor any agency thereof, nor any of their employees, makes any warranty, express or implied, or assumes any legal liability or responsibility for the accuracy, completeness, or usefulness of any information, apparatus, product, or process disclosed, or represents that its use would not infringe privately owned rights. Reference herein to any specific commercial product, process, or service by trade name, trademark, manufacturer, or otherwise does not necessarily constitute or imply its endorsement, recommendation, or favoring by the United States Government or any agency thereof. The views and opinions of authors expressed herein do not necessarily state or reflect those of the United States Government or any agency thereof.
\end{abstract}

Submitted to Cryogenics.

*This work has been sponsored by the U.S. Department of Energy, Energy Efficiency and Renewable Energy, as part of a program to develop electric power technology, under Contract W-31-109-Eng-38. 


\section{DISCLAMMER}

Portions of this document may be illegible in electronic image products. Images are produced from the best available original document. 
Senior author: Dr. Y. S. Cha

Mechanical Engineer

Argonne National Laboratory

Energy Technology Division

Building 335

9700 South Cass Avenue

Argonne, IL 60439 
Running headline: Temperature Distribution and Heating Rate 


\title{
Transverse Temperature Distributions and Heat Generation Rate in Composite Conductors Subjected to a Constant Thermal Disturbance
}

\author{
Y. S. Cha \\ Energy Technology Division \\ Argonne National Laboratory \\ Argonne, Illinois 60439
}

\begin{abstract}
Analytical solution of one-dimensional, transient heat conduction with distributed heat source is obtained to predict the transverse temperature distribution and heat generation rate per unit volume of the composite conductor. The solution indicates that the temperature distribution and the heat generation rate depend on three dimensionless parameters; the dimensionless external disturbance $w_{0}$, the dimensionless interface temperature $\theta^{*}$, and the dimensionless parameter $\phi$ which depends on the thickness and the thermal conductivity of the superconductor. Results of the transient and steady-state solution are presented. It is shown that the heat generation rate per unit volume of the composite $Q / Q_{c}$ is directly proportional to the current in the stabilizer. The dimensionless total internal generation rate $\mathrm{w}_{t}$ in the superconductor is shown to reach a maximum at $\mathrm{Q} / \mathrm{Q}_{\mathrm{c}}=0.5$ because $\mathrm{w}_{\mathrm{t}}$ depends on two competing factors, the current in the superconductor and the electric field strength which is proportional to the current in the stabilizer. The result of the present analysis reduces to a known steady-state solution in the absence of an external disturbance. Limitations of the present analytical model are discussed.
\end{abstract}


Keywords: heat generation, cryogenic stabilization, transient, composite conductors 


\section{Introduction}

Cryogenic stabilization is important for the design and operation of superconducting magnets and is a subject of continuous and ongoing research. Essential to cryogenic stabilization of superconducting magnets is the prediction of heat generation rate per unit volume of the composite conductor. An example is the well known equal-area theory of Maddock, James, and Norris [1]. The heat generation rate per unit volume of the composite conductor is usually calculated by assuming that (1) the current in the superconductor is equal to the critical current with all the excess transferred to the stabilizer, and (2) the critical current decreases linearly with temperature [2]. A more realistic model was proposed by Wilson [2] to calculate the heat generation rate by taking into account of the effects of finite thermal conductivity and thickness of the superconductor. The effect of finite thermal conductivity on heat generation rate is even more pronounced for high-temperature superconductors in view of its relatively low thermal conductivities. The solution reported by Wilson is for steady-state condition and is in the absence of an external disturbance. In this paper, we shall revisit the problem of heat generation rate in a composite conductor by considering the more general case of transient condition and in the presence of a constant thermal disturbance. We treat the problem as one-dimensional and obtain closed-form solution which predicts the transverse temperature distribution in the superconductor and the heat generation rate per unit volume of the composite conductor. It should be noted that most of the papers on stability deal with the propagation of normal zone in the longitudinal direction and assume that the temperature of the composite conductor is uniform in the transverse direction [3-5]. The only exception is the two-dimensional model reported by Chyu and Oberly [6]. However, their solution is numerical and 
therefore does not provide information on what are the relevant dimensionless (scaling) parameters. In this paper, we begin by deriving the governing equations. Then the results of steady-state and transient solutions are presented. Finally, we discuss the limitations of the present model with particular emphasis on its application to high-temperature superconductors.

\section{Analysis}

The objective is to determine the transverse temperature distributions and heat generation rate in composite conductors caused by current redistribution as a result of a constant thermal disturbance. The geometry is shown in Fig. 1 together with the assumed linear relationship between critical current density and temperature. We assume that the characteristic time of current diffusion is short compared to that of thermal diffusion so that the current distribution is completely determined by the temperature distribution [2]. We assume that the length of the thermal disturbance in the axial direction is much larger than the thickness of the superconductor in the transverse direction so that the problem can be treated as one-dimensional in space by neglecting the edge effect. The governing equation in the superconductor is

$$
\frac{1}{\alpha} \frac{\partial \mathrm{T}}{\partial \mathrm{t}}=\frac{\mathrm{W}}{\mathrm{k}}+\frac{\partial^{2} \mathrm{~T}}{\partial \mathrm{X}^{2}}
$$

where $\alpha$ is the thermal diffusivity, $\mathrm{k}$ is the thermal conductivity, $\mathrm{T}$ is temperature, $t$ is time, $X$ is the transverse coordinate, and the heat source per unit volume of the superconductor $\mathrm{W}$ is composed of two parts, 


$$
\mathrm{W}=\mathrm{W}_{0}+\mathrm{W}_{\mathrm{i}}(\mathrm{T})
$$

where $W_{0}$ is the externally imposed heating rate per unit volume of the superconductor and represents a constant thermal disturbance, and $W_{i}(T)$ is the internally generated heating rate per unit volume of the superconductor as a result of current redistribution in the superconductor and the stabilizer. If it is further assumed that the current density in the superconductor is equal to the critical current density corresponding to the local temperature with all the excess current transferred to the stabilizer, then the internal generation rate is [2]

$$
W_{i}(T)=E J(T)=E J_{c}(T)=E J_{c}\left(T_{i}\right)\left(T_{c}-T\right) /\left(T_{c}-T_{i}\right)
$$

where $T_{i}$ is the temperature at the interface of the superconductor and the stabilizer, $\mathrm{J}$ is current density, $\mathrm{J}_{c}$ is the critical current density, $\mathrm{T}_{c}$ is the critical temperature of the superconductor, and $\mathrm{E}$ is the electrical field strength developed in the composite conductor as a result of current flow in the stabilizer. $E$ is assumed to be uniform across the entire conductor. The initial and boundary conditions are

$$
\begin{gathered}
\mathrm{t}=0, \mathrm{~T}=\mathrm{T}_{\mathrm{i}}, \\
\mathrm{X}=0, \frac{\partial \mathrm{T}}{\partial \mathrm{X}}=0, \text { and } \\
\mathrm{X}=\mathrm{a}, \mathrm{T}=\mathrm{T}_{\mathrm{i}} .
\end{gathered}
$$

If we assume that the thermal conductivity of the metal stabilizer is much larger than that of the superconductor (which is applicable to high-temperature ceramic 
superconductors) and that the thickness of the superconductor (a) is not small compared to that of the stabilizer (b), then we do not need to solve the heat conduction equation in the stabilizer. Substituting Eqs. 2 and 3 into Eq. 1 and the resulting equation can be solved by Laplace transform [7]. After some manipulation, the solution turns out to be

$$
\begin{aligned}
\theta= & {\left[\frac{\cosh (\beta \mathrm{x})}{\cosh (\beta)}\right]\left(1+\frac{\mathrm{w}_{0} \theta_{\mathrm{g}}}{\mathrm{Q} / \mathrm{Q}_{\mathrm{c}}}\right)-\frac{\mathrm{w}_{0} \theta_{\mathrm{g}}}{\mathrm{Q} / \mathrm{Q}_{\mathrm{c}}} } \\
& +2\left(\mathrm{w}_{0} \phi \theta_{\mathrm{g}}+\beta^{2}\right) \sum_{\mathrm{n}=0}^{\infty} \frac{(-1)^{\mathrm{n}} \exp \left[-\left(\mathrm{N}^{2}+\beta^{2}\right) \tau\right] \cos (\mathrm{Nx})}{\mathrm{N}\left(\beta^{2}+\mathrm{N}^{2}\right)}
\end{aligned}
$$

where $N=(2 n+1) \pi / 2$ and the dimensionless variables are defined as,

$$
\begin{gathered}
\theta=\left(\mathrm{T}_{\mathrm{c}}-\mathrm{T}\right) /\left(\mathrm{T}_{\mathrm{c}}-\mathrm{T}_{\mathrm{i}}\right), \\
\mathrm{x}=\mathrm{X} / \mathrm{a}, \\
\tau=\alpha t / \mathrm{a}^{2}, \\
\beta^{2}=\mathrm{E} J_{c}\left(\mathrm{~T}_{\mathrm{i}}\right) \mathrm{a}^{2} /\left[\mathrm{k}\left(\mathrm{T}_{\mathrm{c}}-\mathrm{T}_{\mathrm{j}}\right)\right], \\
\theta_{\mathrm{g}}=\left(\mathrm{T}_{\mathrm{c}}-\mathrm{T}_{\mathrm{g}}\right) /\left(\mathrm{T}_{\mathrm{c}}-\mathrm{T}_{\mathrm{i}}\right),
\end{gathered}
$$

and 


$$
\mathrm{w}_{0}=\mathrm{W}_{0} \lambda / \mathrm{Q}_{\mathrm{c}}
$$

The parameter $\lambda=a /(a+b)$ is the superconductor fraction in the composite and the critical generation rate $Q_{c}$ (the heat generation rate per unit volume of the composite when most of the current is in the stabilizer) is defined as

$$
\mathrm{Q}_{\mathrm{c}}=\rho \mathrm{J}_{0}^{2}\left(\mathrm{~T}_{0}\right) \lambda^{2} /(1-\lambda)
$$

where $\rho$ is the electrical resistivity of the stabilizer. The parameter Q in Eq. 6 is the internal heat generation rate per unit volume of the composite conductor (superconductor plus stabilizer), which is unknown and needs to be determined. The parameter $Q$ can be expressed in terms of the electric field strength $E$ and the current density $\mathrm{J}_{0}\left(\mathrm{~T}_{0}\right)$,

$$
\mathrm{Q}=\lambda \mathrm{E} \mathrm{J}_{0}\left(\mathrm{~T}_{0}\right)
$$

Using Eqs. 10, 13, and 14, it can be shown that

$$
\beta^{2}=\left(Q / Q_{c}\right) \phi,
$$

where

$$
\phi=Q_{c} a(a+b) /\left[k\left(T_{c}-T_{g}\right)\right] .
$$

There is an additional condition that must be satisfied as a result of current redistribution, which is that the sum of the current in the superconductor and the stabilizer must be equal to the total current, 


$$
\int_{0}^{a} J_{c}(T) d X+b E / \rho=a J_{0}\left(T_{0}\right)
$$

Employing the dimensionless parameters defined previously, it can be shown that Eq. 17 is equivalent to

$$
\int_{0}^{1} \theta d x+\left(Q / Q_{c}\right) \theta_{g}=\theta_{g}
$$

Substituting Eq. 6 into Eq. 18 resulted in the following relationship between $\theta_{\mathrm{g}}$ and $\mathrm{Q} / \mathrm{Q}_{\mathrm{c}}$

$$
\theta_{g}=\frac{\frac{\tanh (\beta)}{\beta}+S}{\left(1-Q / Q_{c}\right)+\left[1-\frac{\tanh (\beta)}{\beta}\right]\left(\frac{w_{0}}{Q / Q_{c}}\right)-2 w_{0} \phi S}
$$

where

$$
S=\sum_{n=0}^{\infty} \frac{(-1)^{n} \exp \left[-\left(N^{2}+\beta^{2}\right) \tau\right] \sin (N)}{\left(\beta^{2}+N^{2}\right) N^{2}}
$$

We have now completed the formulation of the problem. For given values of $\mathrm{w}_{0}$, $\theta_{g}$, and $\phi$, we want to calculate $\theta$ as functions of $\tau$ and $x$ and $Q / Q_{c}$ and $\beta$ as function $\tau$ by solving Eqs. 6, 15, and 19 simultaneously. Numerically, it is easier to carry out the calculations in a slightly different manner. Instead of $w_{0}, \theta_{g}$, and $\phi$, one can use $\mathrm{w}_{0}, Q / \mathrm{Q}_{\mathrm{c}}$, and $\phi$ as inputs, then $\beta$ can be calculated explicitly from Eq. 15. Then $\theta_{\mathrm{g}}$ can be calculated by using Eq. 19. Finally, the 
dimensionless temperature $\theta$ is calculated by using Eq. 6. The calculations are straightforward because no iteration is involved.

There is a simple relationship between the heat generation rate per unit volume of the composite and the current in the stabilizer $\left(\mathrm{I}_{\mathrm{st}}\right)$. Substituting the electric field strength $\mathrm{E}=\rho \mathrm{J}_{\text {st }}$ into Eq. 14, it can be shown that

$$
\begin{gathered}
\mathrm{J}_{\mathrm{st}} / \mathrm{J}_{0}\left(\mathrm{~T}_{0}\right)=\left(\mathrm{Q} / \mathrm{Q}_{\mathrm{c}}\right) \mathrm{a} / \mathrm{b}, \text { or } \\
\mathrm{I}_{\mathrm{st}} / \mathrm{I}_{0}=\mathrm{Q} / \mathrm{Q}_{\mathrm{c}} .
\end{gathered}
$$

Thus, the fraction of current in the stabilizer is equal to the fraction of the heat generated in the composite conductor. Another interesting parameter is the total internal heat generated per unit volume in the superconductor,

$$
\mathrm{W}_{\mathrm{t}}=\int_{0}^{\mathrm{a}} \mathrm{W}_{\mathrm{i}}(\mathrm{T}) \mathrm{dX} / \mathrm{a}
$$

Since the total heat generated in the composite is equal to the sum of the heat generated in the superconductor and the stabilizer, it follows that

$$
Q(a+b)=W_{t} a+\rho\left(J_{s t}\right)^{2} b
$$

Substituting Eq. 21 into Eq. 23 and solve for the dimensionless parameter $\mathrm{w}_{t}$,

$$
\mathrm{w}_{\mathrm{t}}=\mathrm{W}_{\mathrm{t}} \lambda / \mathrm{Q}_{\mathrm{c}}=\left(\mathrm{Q} / \mathrm{Q}_{\mathrm{c}}\right)\left(1-\mathrm{Q} / \mathrm{Q}_{\mathrm{c}}\right)
$$


Both Eqs. 21 and 24 appear to be quite simple and depend only on the heat generation rate per unit volume of the composite $Q / Q_{c}$. However, because $Q / Q_{c}$ depends on $\mathrm{w}_{0}, \phi$, and $\theta_{\mathrm{g}}$, so do $\mathrm{I}_{\mathrm{st}} / \mathrm{I}_{0}$ and $\mathrm{w}_{\mathrm{t}}$. Equations 21 and 24 are applicable to both the transient and steady-state situations.

Before presenting the results, it is interesting to point out a special case of the general solution given by Eq. 6. Since the argument in the exponential term is negative, a steady-state solution always exist as $\tau$ becomes large. Under steadystate condition, Eqs. 6 and 19 are reduced to

$$
\begin{gathered}
\theta=\left[\frac{\cosh (\beta x)}{\cosh (\beta)}\right]\left(1+\frac{\mathrm{w}_{0} \theta_{\mathrm{g}}}{\mathrm{Q} / \mathrm{Q}_{\mathrm{c}}}\right)-\frac{\mathrm{w}_{0} \theta_{\mathrm{g}}}{\mathrm{Q} / \mathrm{Q}_{\mathrm{c}}} \\
\theta_{\mathrm{g}}=\frac{\tanh (\beta) / \beta}{\left(1-\mathrm{Q} / \mathrm{Q}_{\mathrm{c}}\right)+[1-\tanh (\beta) / \beta]\left(\frac{\mathrm{w}_{0}}{\mathrm{Q} / \mathrm{Q}_{\mathrm{c}}}\right)} .
\end{gathered}
$$

If there is no external thermal disturbance $\left(\mathrm{w}_{0}=0\right)$, then the steady-state solution becomes

$$
\begin{gathered}
\theta=\cosh (\beta \mathrm{x}) / \cosh (\beta), \\
\theta_{\mathrm{g}}=\tanh (\beta) / \beta /\left(1-\mathrm{Q} / \mathrm{Q}_{\mathrm{c}}\right) .
\end{gathered}
$$

Equations 27 and 28 are identical to the solution reported by Wilson [2], who calculated the steady-state, transverse temperature distributions and heat generation rate and applied the results to determine the cryogenic stability of a composite conductor. 


\section{Results and Discussions}

The results of the previous analysis will be presented in two parts. The first part describes the numerical results of the steady-state solution and the second part describes the results of the transient solution. Instead of $\theta_{\mathrm{g}}$, we will define a dimensionless parameter $\theta^{*}$

$$
\theta^{*}=\left(T_{i}-T_{g}\right) /\left(T_{c}-T_{g}\right)
$$

The relation between $\theta_{\mathrm{g}}$ and $\theta^{*}$ is

$$
\theta^{*}=1-1 / \theta_{g}
$$

The results will be expressed in terms of $\theta^{*}$, which is the parameter employed by Wilson [2], so that a direct comparison can be made.

\section{Steady-State Solution}

In this case, the exponential terms in Eqs. 6 and 19 approach zero as $\tau$ becomes very large. Figures 2 to 4 show the calculated $\theta^{*}$ as a function of $Q / Q_{c}$ (or $\left.I_{s t} / I_{0}\right)$ for various values of $\phi$. When there is no external disturbance $\left(w_{0}=0\right)$, the result is shown in Fig. 2 and it is identical to that reported by Wilson [2]. The case of $\phi=0$ corresponds to the special situation where the superconductor is at a uniform temperature because the thermal conductivity $\mathrm{k}$ approaches infinity. It can be seen from Fig. 2 that $\theta^{*}=0\left(T_{i}=T_{g}\right)$ at $I_{s t} / I_{0}=0$ and $\theta^{*}=1\left(T_{i}=T_{c}\right)$ at $I_{s t} / I_{0}=$ 1. This means that, in the absence of a external disturbance, all the current is in 
the superconductor when $T_{i}=T_{g}$, and all the current is in the stabilizer when $T_{i}=$ $T_{c}$. The situation is different when there is an external disturbance $\left(w_{0}>0\right)$ as shown in Figs. 3 and 4. Except for $\phi=0$, it can be observed that $\theta^{*}<0\left(T_{i}<T_{g}\right)$ when $\mathrm{I}_{\text {st }} / \mathrm{I}_{0}=0$ and $\theta^{*}<1\left(\mathrm{~T}_{\mathrm{i}}<\mathrm{T}_{\mathrm{c}}\right)$ when $\mathrm{I}_{\mathrm{st}} / \mathrm{I}_{0}=1$. In order to maintain all the current in the superconductor in the presence of an external disturbance, the interface temperature $T_{i}$ must be smaller than the generation temperature $T_{g}$. This is because the external disturbance causes current redistribution within the superconductor even though all the current is still in the superconductor. On the other hand, when all the current is transferred to the stabilizer, Figs. 3 and 4 show that the interface temperature $T_{i}$ is less than the critical temperature $T_{c}$. This result is incorrect physically because if the interface temperature is less than the critical temperature, then a portion of the superconductor (near the interface) is still capable of carrying a certain amount of current and consequently not all the current is in the stabilizer. This unrealistic result arises because the analytical model could not account for the situation when the temperature of the superconductor exceeds the critical temperature. This can be explained by examining Eq. 3 which is the assumed internal generation rate in the superconductor. When $\mathrm{T}$ is greater than $\mathrm{T}_{\mathrm{c}}$, the internal generation rate becomes negative which is obviously incorrect. When $T_{i}$ is slightly smaller or equal to $T_{c}$ at $I_{s t} / I_{0}=1$ as shown in Figs. 2 to 4 , the bulk of the superconductor is at temperatures greater than $\mathrm{T}_{\mathrm{c}}$ and Eq. 3 fails to describe the physical situation correctly. We shall discuss this further in a later section.

Another important feature which can be observed from Figs. 2 to 4 is that when $\phi>3$, a relative minimum appears in each of the curve in these figures. For example, when $\phi=5, Q / Q_{c}$ increases with decreasing $\theta^{*}$ for $Q / Q_{c}<1.5$ and increase with increasing $\theta^{*}$ for $Q / Q_{c}>1.5$. The portion of the curve with negative 
slope is most likely unstable because as the interface temperature $T_{i}$ is reduced, the superconductor should be able to carry more current and therefore less current is transferred to the stabilizer.

Typical temperature distribution in the superconductor is shown in Fig. 5 where the dimensionless temperature $\theta$ is plotted against $\mathrm{x}$ for various values of $\mathrm{Q} / \mathrm{Q}_{\mathrm{c}}$ (or $\mathrm{I}_{\mathrm{st}} / \mathrm{I}_{0}$ ) with $\mathrm{w}_{0}=0.2$ and $\phi=5$. As $\mathrm{Q} / \mathrm{Q}_{\mathrm{c}}$ is increased, the temperatures in the core of the superconductor increases and approaches $T_{c}(\theta=0)$. However, when $Q / Q_{c}$ is greater than $0.8, \theta$ becomes negative and $T$ becomes greater $T_{c}$. This is again the result of employing Eq. 3 which is not valid when the temperature of the superconductor exceeds the critical temperature.

It is interesting to find out how the dimensionless total internal generation rate $w_{t}$ varies with the dimensionless temperature $\theta^{*}$ and the heat generation rate per unit volume of the composite $Q / Q_{c}$. Figure 6 shows the plot of $w_{t}$ versus $\mathrm{Q} / \mathrm{Q}_{\mathrm{c}}$ (or $\mathrm{I}_{\mathrm{st}} / \mathrm{I}_{0}$ ) calculated by Eq. 24. The total internal generation reaches a maximum at $I_{s t} / I_{0}=0.5$. The reason that $w_{t}$ has a maximum is because the internal generation rate depends on the product of two competing factors, the total current in the superconductor and the electric field strength. When the total current in the superconductor is large, the electric field strength is small because the current in the stabilizer is small. When the total current in the superconductor is small, the electric field strength is large because the current in the stabilizer is large. This characteristic is clearly demonstrated in Fig. 6 and by Eq. 24. Figures 7 and 8 show the variation of $\theta^{*}$ with $w_{t}$ for various values of $\phi$. Again, $w_{t}$ reaches a maximum at some value of $\theta^{*}$ which depends on the values of $\phi$ and $\mathrm{w}_{0}$. 


\section{Transient Solution}

Figures 9 and 10 show the results of the transient solution calculated by Eqs. 19 and 30. It appears that the steady-state is approached when $\tau \cong 1$. This means that the time required to reach steady-state is $t \cong a^{2} / \alpha$. This characteristic time for transverse heat conduction in the superconductor can be compared to that of current diffusion in the stabilizer. The characteristic time of current diffusion in the stabilizer is $t_{s t} \cong \mu_{0} \mathrm{~L}^{2} / \rho$, where $\mu_{0}\left(=4 \pi \times 10^{-7} \mathrm{H} / \mathrm{m}\right)$ is the permeability of free space, and $L$ is the characteristic length in the longitudinal direction. The ratio of the two characteristic times is

$$
t / t_{s t}=\rho a^{2} / \alpha \mu_{0} L^{2}
$$

The thermal diffusivity for high-temperature superconductors at $77 \mathrm{~K}$ is $\alpha \cong 10^{-6}$. $10^{-7} \mathrm{~m}^{2} / \mathrm{s}$, and the resistivity of silver at $77 \mathrm{~K}$ is $\rho \cong 3.65 \times 10^{-9} \Omega-\mathrm{m}$. In the analytical model, we assumed that $L$ is much larger than a so that edge effect can be neglected and the problem can be treated as one-dimensional. If $\mathrm{L} / \mathrm{a}=10$,

$$
\mathrm{t} / \mathrm{t}_{\mathrm{st}} \cong 0.58 \times 10^{2}>>1
$$

Then the time required for current redistribution in the stabilizer is much shorter then that required for the transverse temperature in the superconductor to reach steady-state. Steady-state current in the stabilizer and the electric field strength can be assumed to establish instantly. But, as one can see that the margin is relatively small. If $L / a>10$ is required, then $t / t_{s t}$ will no longer be much greater than 1 , and the error introduced by the one-dimensional model will increase. 


\section{Summary}

Closed-form solution of transient heat conduction with distributed heat source is employed to predict the transverse temperature distribution and heat generation rate per unit volume of a composite conductor subjected to a constant thermal disturbance. The heat source includes a constant external disturbance and an internal generation term which is a function of temperature distribution in the superconductor (Eq. 3). It is shown that the dimensionless temperature distribution $\theta$ (Eq. 6) and the heat generation rate per unit volume of the composite $\mathrm{Q} / \mathrm{Q}_{\mathrm{c}}$ depend on the three dimensionless parameters $\theta_{\mathrm{g}}, \phi$, and $\mathrm{w}_{0}$. Results of the transient and steady-state solution are presented in Fig. 2 to 10. It is shown that the heat generation rate per unit volume of the composite $Q / Q_{c}$ is directly proportional to the current in the stabilizer (Eq. 21). The dimensionless total internal generation rate $w_{t}$ in the superconductor is shown to reach a maximum at $Q / Q_{c}=0.5$ (Fig. 6) because $w_{t}$ depends on two competing factors, the current in the superconductor and the electric field strength which is proportional to the current in the stabilizer. It is demonstrated that steady-state is approached when the dimensionless time $\tau$ becomes greater than 1 . In the absence of an external disturbance, the steady-state solution reduces to that reported by Wilson [2].

One of the basic assumption made in the present analysis is that the characteristic time of current redistribution in the superconductor and the stabilizer is much smaller than that of temperature redistribution so that heat conduction in the superconductor is independent of the current diffusion process in the stabilizer. This assumption is shown to be marginal for high-temperature superconductors. 
Another basic assumption made in the present analysis is the distribution of internal heat generation rate described by Eq. 3. It is evident that Eq. 3 cannot accommodate temperature greater than $T_{c}$ because dissipation becomes negative when $\mathrm{T}>\mathrm{T}_{\mathrm{c}}$ and Eq. 3 gives unrealistic results. In reality, $\mathrm{T}$ can be greater than $\mathrm{T}_{\mathrm{c}}$ and the local current density should be reduced to zero if $\mathrm{T}>\mathrm{T}_{\mathrm{c}}$. Consequently, the local heat generation rate should be zero. In other words, $W_{i}=0$ and $J=0$ when $\mathrm{T}>\mathrm{T}_{\mathrm{c}}$. This additional constraint will cause complication for analytical solution but it can be easily handled by numerical solution. We are currently carrying out the numerical solution. In the numerical solution, we can also include the more general condition of a time dependent external disturbance instead of a constant external disturbance used in the present analysis. The present analytical solution can be employed to validate the results of the numerical solution under special circumstances.

\section{Acknowledgments}

This work has been supported by the U.S. Department of Energy, Energy Efficiency and Renewable Energy, as part of a program to develop electric power technology, under Contract W-31-109-Eng-38.

\section{References}

1. B. J. Maddock, G. B. James, and W. T. Norris, "Superconductive Composites: Heat Transfer and Steady State Stabilization", Cryogenics, 9, 261-273, 1969. 
2. M. N. Wilson, Superconducting Magnets, Oxford University Press, New York, Chap. 6, 1983.

3. V. R. Romanovskii, "Stability of Superconducting Composites under Thermal Disturbances with Change in the External Magnetic Field and the Critical Temperature of the Superconductor", Cryogenics, 28, 756-761, 1988.

4. A. Abeln, E. Klemt, and H. Reiss, "Stability Considerations for Design of a High Temperature Superconductor", Cryogenics, 32, 269-278, 1992.

5. R. Kupferman, R. G. Mints, and E. Ben-Jacob, "Propagating Normal Domains in Large Composite Superconductors", J. Appl. Phys. 70 (12), 74847491, Dec. 1991.

6. M. K. Chyu and C. E. Oberly, "Effects of Transverse Heat Transfer on Normal Zone Propagation in Metal-Clad High Temperature Superconductor Coil Tape", Cryogenics, 31, 680-686, 1991.

7. H. S. Carslaw and J. C. Jaeger, Conduction of Heat in Solids, Oxford University Press, 2nd Ed., 1980, pp. 404-405.

\section{Nomenclature}

a $\quad 1 / 2$ the thickness of the superconductor, $m$

b thickness of the stabilizer, $\mathrm{m}$

E electric field strength, V/m

I current, A 


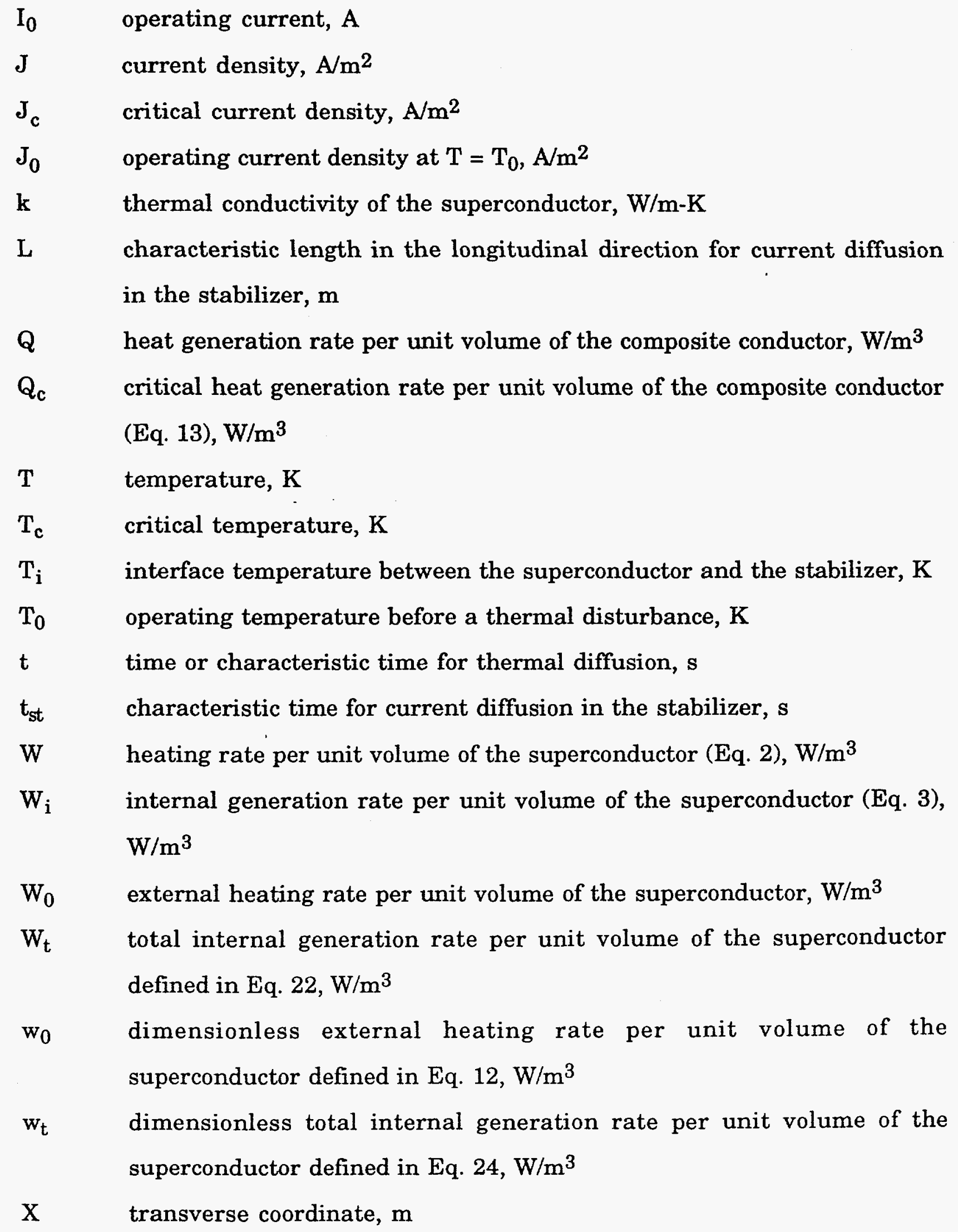


$\mathrm{x}$ dimensionless transverse coordinate, $\mathrm{X} / \mathrm{a}$

$\alpha \quad$ thermal diffusivity of the superconductor, $\mathrm{m}^{2} / \mathrm{s}$

$\beta \quad$ dimensionless parameter defined in Eq. 10

$\rho$ electrical resistivity of the stabilizer, $\Omega-m$

$\lambda$ fraction of superconductor in the composite, $a /(a+b)$

$\phi \quad$ a dimensionless parameter defined in Eq. 16.

$\tau$ dimensionless time, $\alpha \mathrm{t} / \mathrm{a}^{2}$

$\theta \quad$ dimensionless temperature defined in Eq. 7

$\theta_{\mathrm{g}}$ dimensionless temperature defined in Eq. 11

$\theta^{*} \quad$ dimensionless temperature defined in Eq. 30

\section{Subscripts}

st

stabilizer 


\section{Figure Captions}

Fig. 1 A schematic of the geometry of the model and the assumed relationships between the critical current density and the temperature.

Fig. 2 Variation of $\theta^{*}$ with $Q / Q_{c}$ or $I_{s t} / I_{0}$ for various values of $\phi$ with $w_{0}=0$ under steady-state condition.

Fig. 3 Variation of $\theta^{*}$ with $Q / Q_{c}$ or $I_{s t} / I_{0}$ for various values of $\phi$ with $w_{0}=0.1$ under steady-state condition.

Fig. 4 Variation of $\theta^{*}$ with $Q / Q_{c}$ or $I_{s t} / I_{0}$ for various values of $\phi$ with $w_{0}=0.2$ under steady-state condition.

Fig. 5 Variation of $\theta$ with $x$ for various values of $Q / Q_{c}$ or $I_{s t} / I_{0}$ with $w_{0}=0$ and $\phi=5$ under steady-state condition.

Fig. 6 Variation of $\mathrm{w}_{\mathrm{t}}$ with $\mathrm{Q} / \mathrm{Q}_{\mathrm{c}}$ or $\mathrm{I}_{\mathrm{st}} / \mathrm{I}_{0}$.

Fig. 7 Variation of $\theta^{*}$ with $\mathrm{w}_{\mathrm{t}}$ for various values of $\phi$ with $\mathrm{w}_{0}=0$.

Fig. 8 Variation of $\theta^{*}$ with $\mathrm{w}_{\mathrm{t}}$ for various values of $\phi$ with $\mathrm{w}_{0}=0.1$.

Fig. 9 Variation of $\theta^{*}$ with $Q / Q_{c}$ or $I_{s t} / I_{0}$ with $w_{0}=0$ and $\phi=5$ under transient condition. 
Fig. 10 Variation of $\theta^{*}$ with $Q / Q_{c}$ or $I_{s t} / I_{0}$ with $w_{0}=0.1$ and $\phi=5$ under transient condition. 


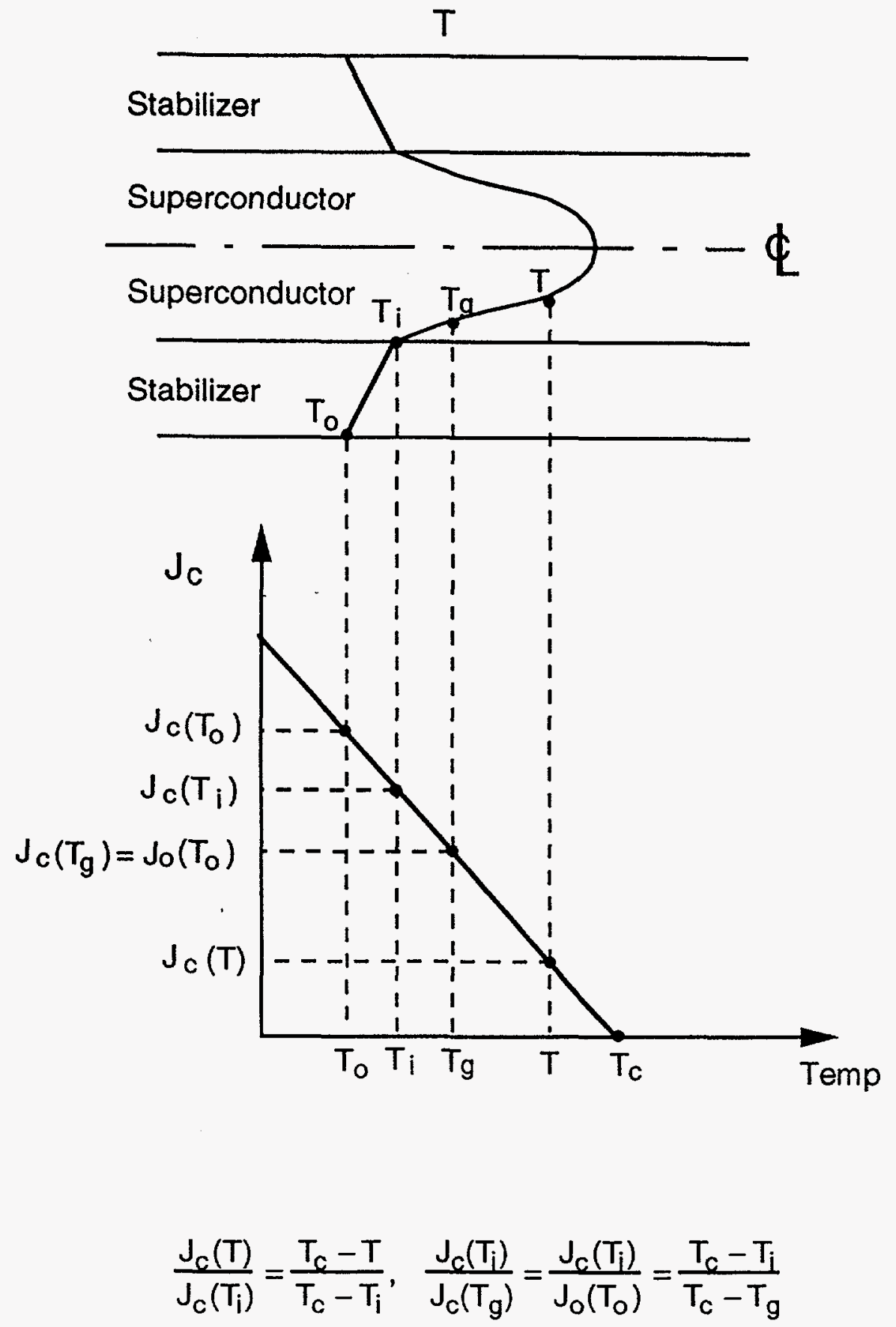

Fig.l 


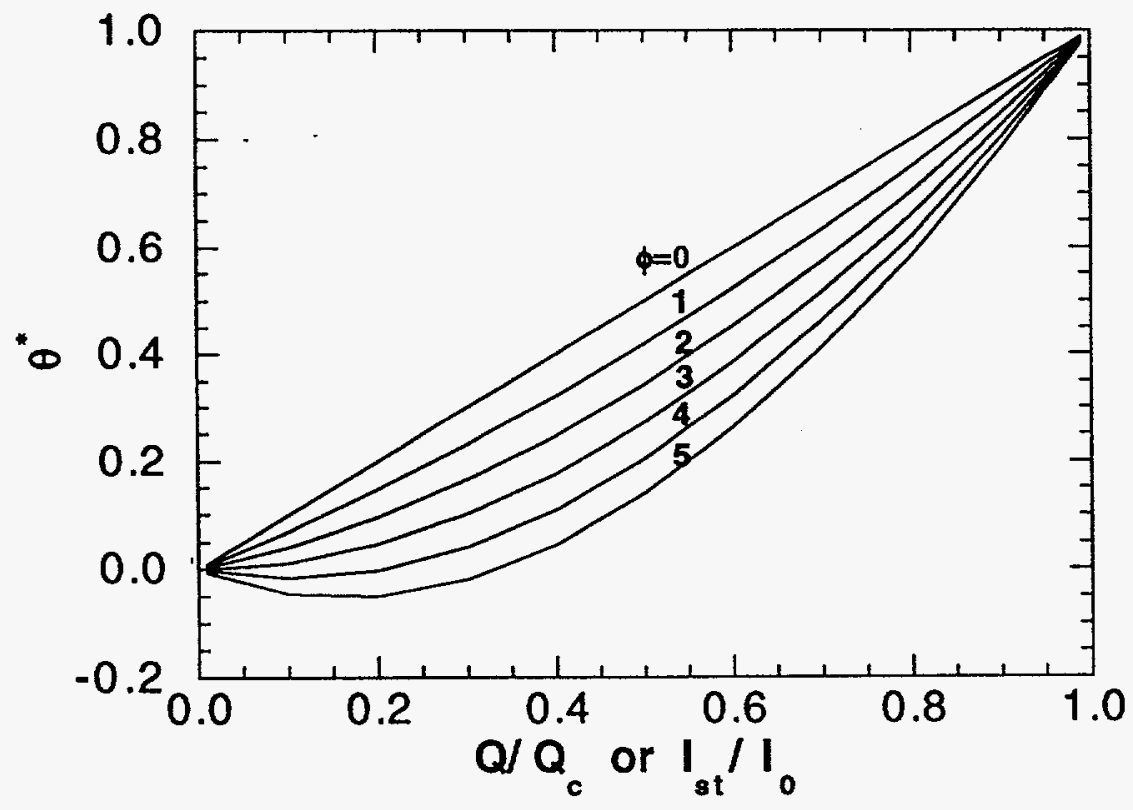




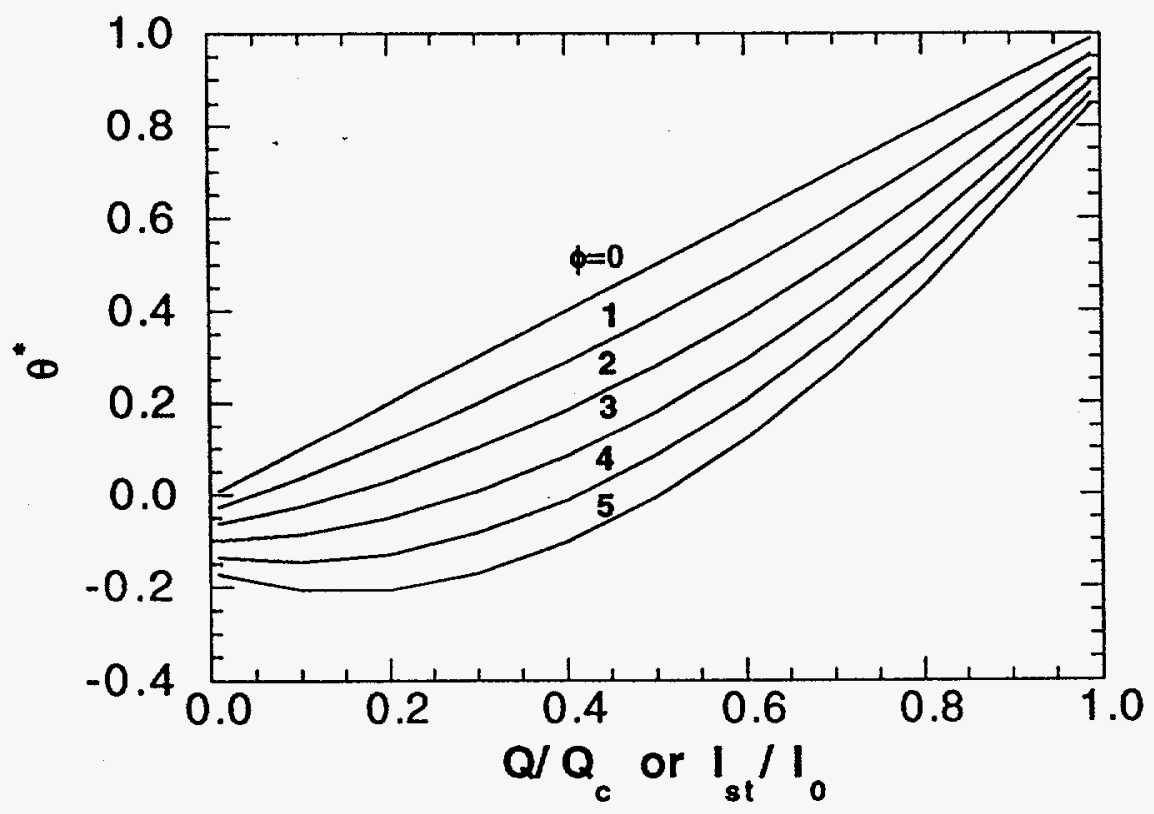

Fig. 3 


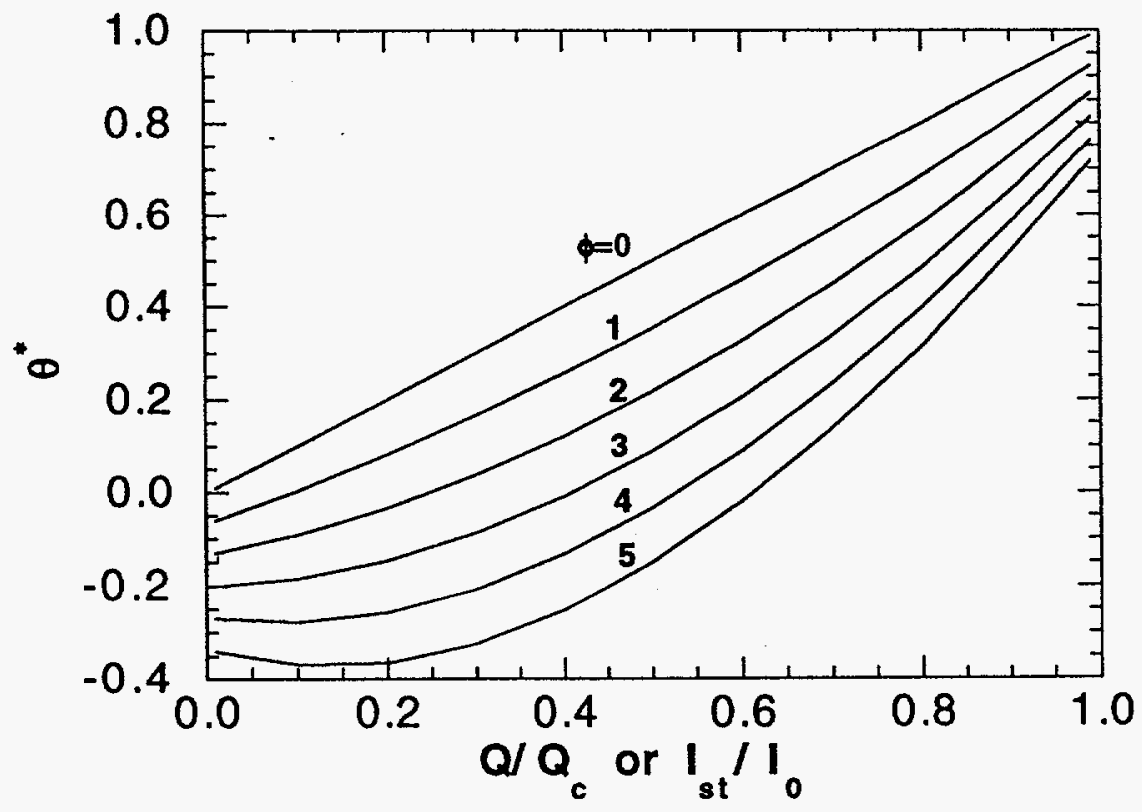

Fig 4 


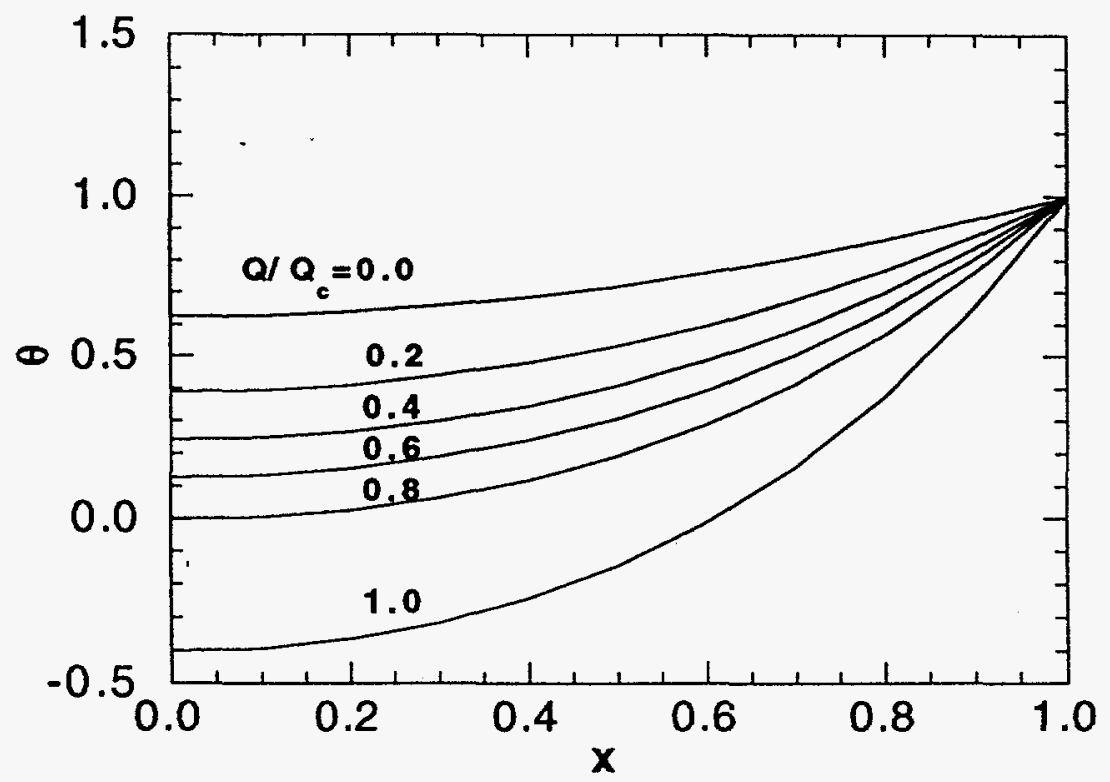

Fig. 5 


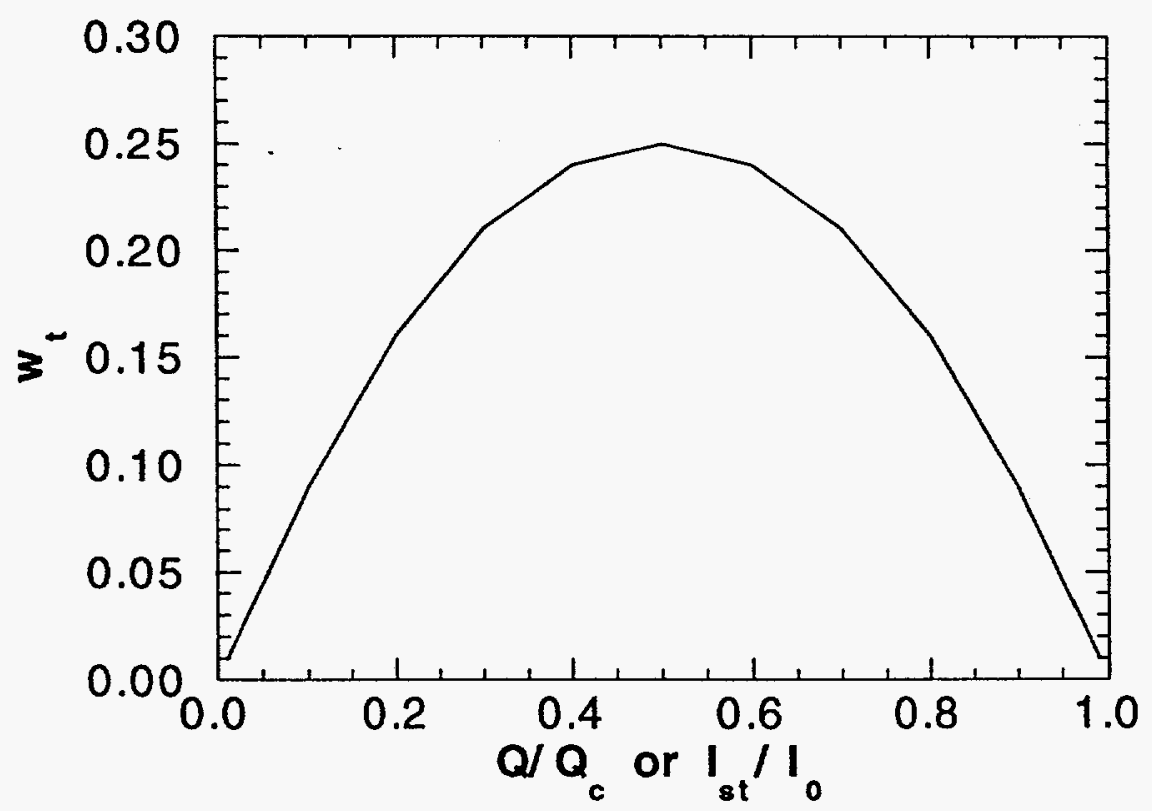

Fig. 6 


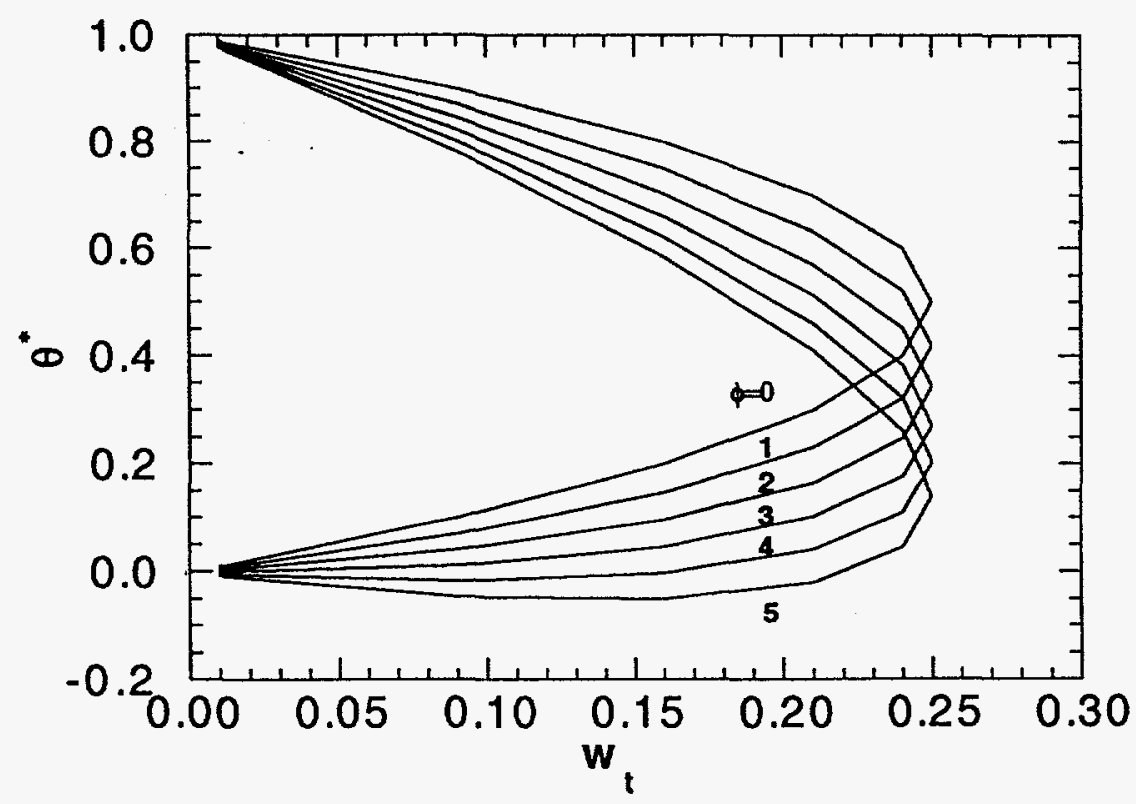

Fig.7 


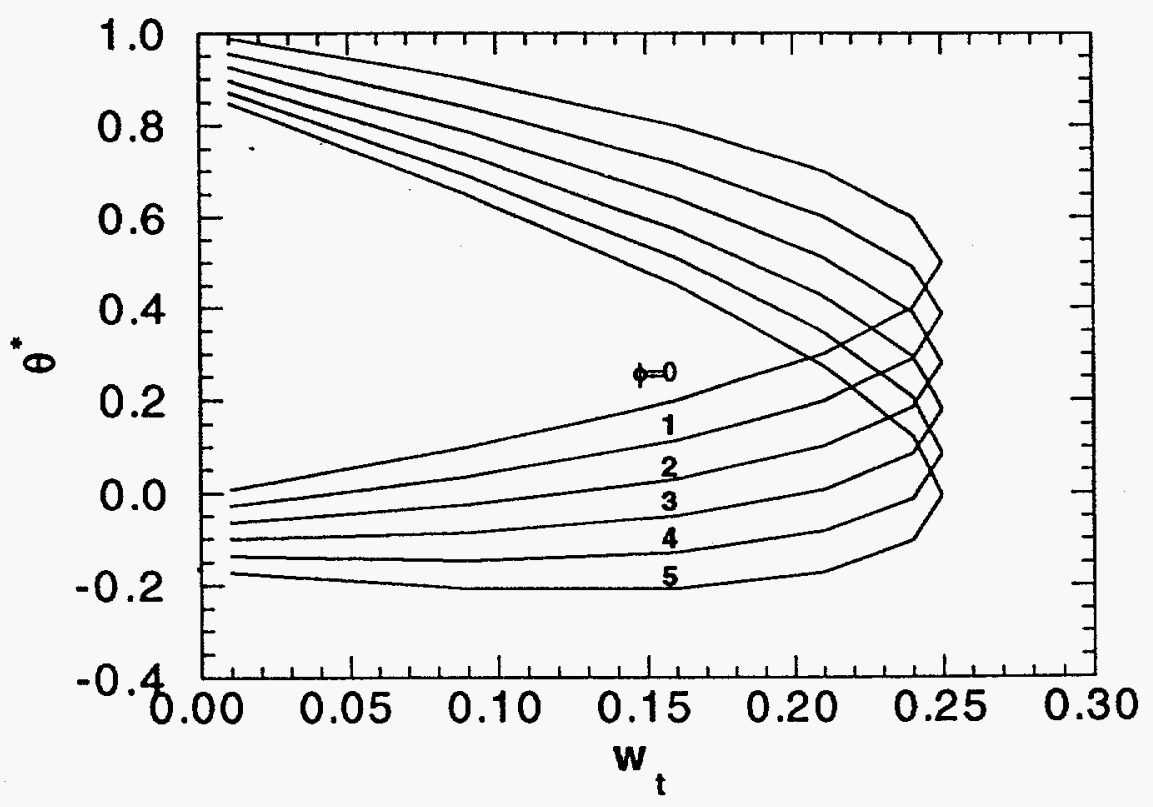

Fig. 8 


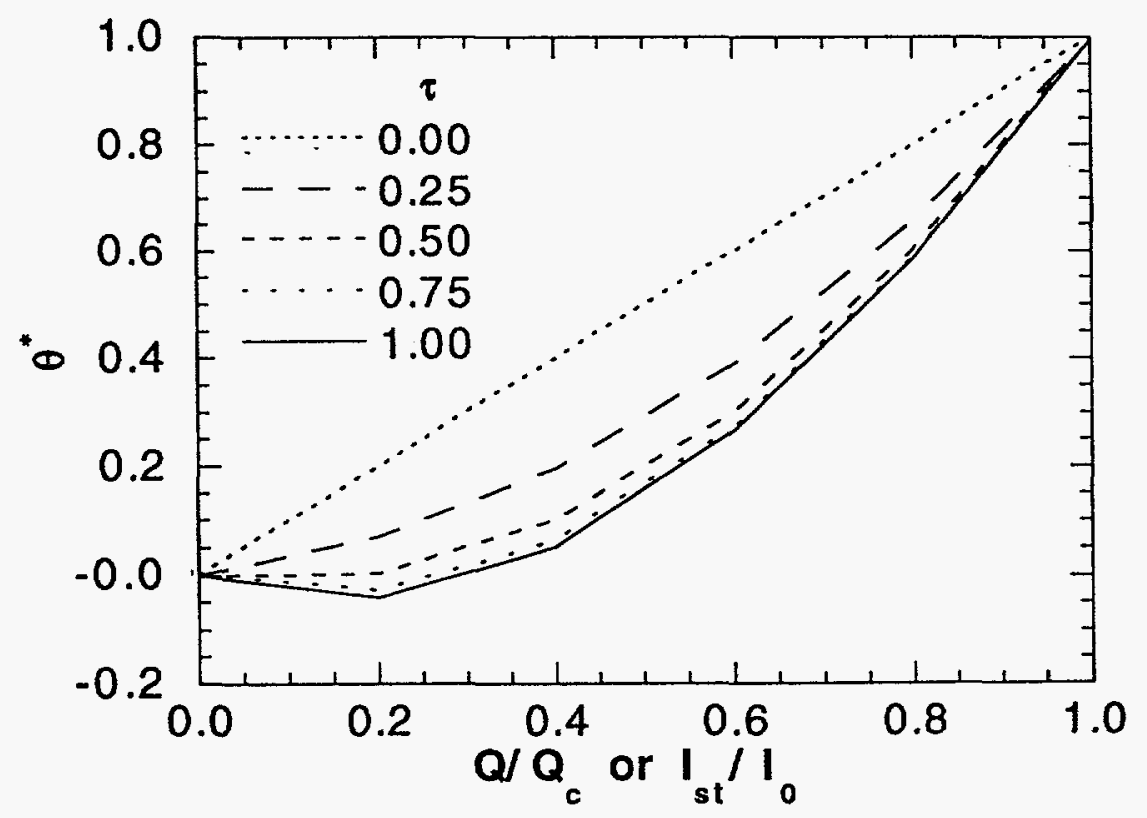

Fig. 9 


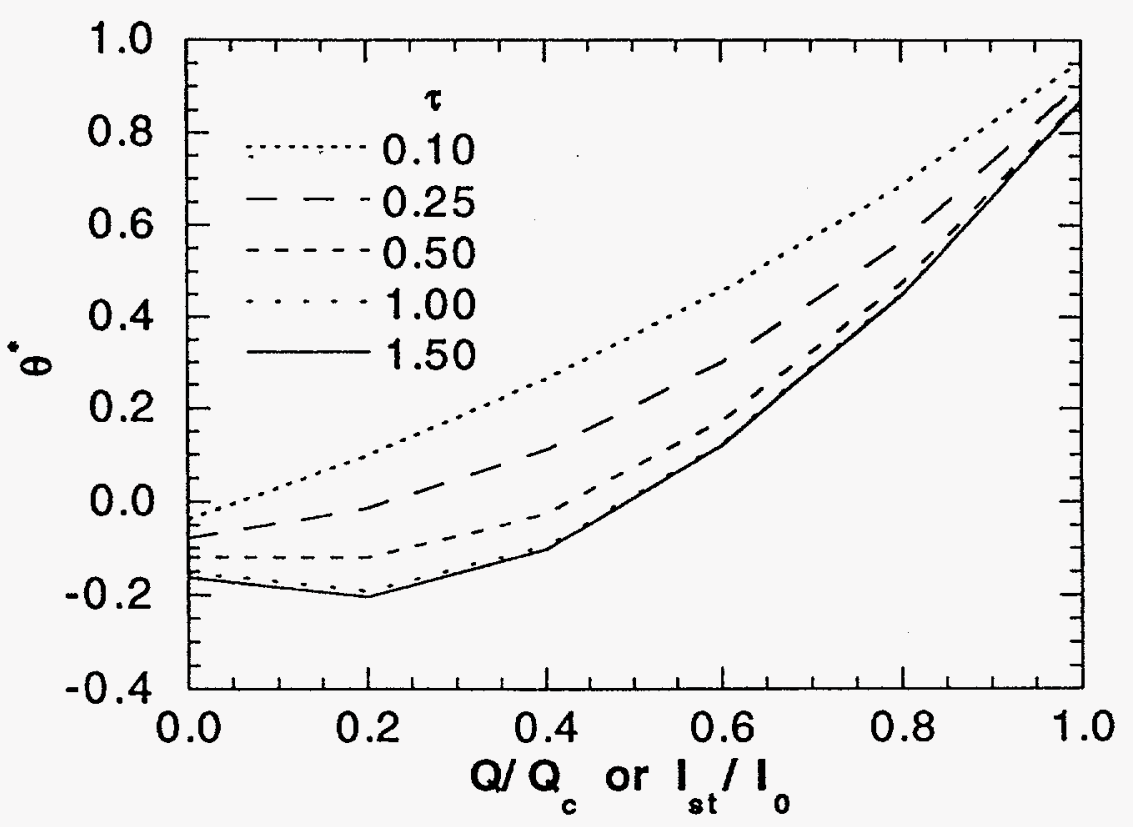

Fig. 10 